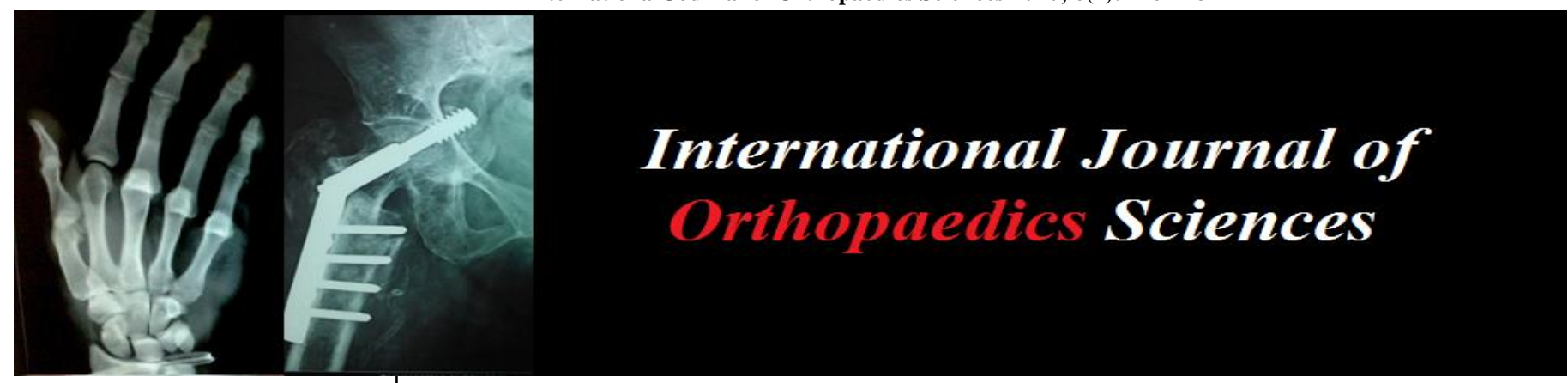

E-ISSN: 2395-1958

P-ISSN: 2706-6630

IJOS 2020; 6(2): 476-478

(C) 2020 IJOS

www.orthopaper.com

Received: 10-02-2020

Accepted: 12-03-2020

Dr. Jairam D Jagiasi

Additional Professor

Department of Orthopaedics,

Dr. R.N. Cooper hospital and

H.B.T. Medical College,

Vile Parle (W), Mumbai,

Maharashtra, India

Dr. Parth Mehta

Senior registrar,

Department of Orthopaedics,

Dr. R.N. Cooper hospital and

H.B.T. Medical College,

Vile Parle (W), Mumbai,

Maharashtra, India

Dr. Tushar V Ubale

Assistant Professor,

Department of Orthopaedics,

Dr. R.N. Cooper hospital and

H.B.T. Medical College,

Vile Parle (W), Mumbai,

Maharashtra, India

Dr. Mohit R Upadhyaya Specialty Medical Officer, Department of Orthopaedics, Dr. R.N. Cooper hospital and H.B.T. Medical College, Vile Parle (W), Mumbai, Maharashtra, India

Dr. Ruchi Prasad

Intern, Department of Orthopaedics, Dr. R.N. Cooper hospital and H.B.T. Medical College, Vile Parle (W), Mumbai,

Maharashtra, India
Corresponding Author: Dr. Parth Mehta Senior registrar, Department of Orthopaedics, Dr. R.N. Cooper hospital and H.B.T. Medical College, Vile Parle (W), Mumbai, Maharashtra, India

\section{How expert is genexpert test: A study done at a tertiary care centre for TB spine patients}

\author{
Dr. Jairam D Jagiasi, Dr. Parth Mehta, Dr. Tushar V Ubale, Dr. Mohit R \\ Upadhyaya and Dr. Ruchi Prasad
}

DOI: https://doi.org/10.22271/ortho.2020.v6.i2h.2085

\section{Abstract}

Study design: Retrospective study.

Purpose: The aim of this study was to analyze various diagnostic tools, including Gene Xpert, for the management of tuberculosis of the spine.

Aim of study: The aim of this study was to analyze various diagnostic tools for the management of tuberculosis of the spine, particularly the use of Gene Xpert, and to compare it with other diagnostic tools.

Inclusion criteria: All cases which had at least one positive test out of Gene Xpert, smear, culture sensitivity and histopathology

Exclusion criteria: All cases which tested negative for Gene Xpert, smear, culture sensitivity and histopathology

Materials and methods: A Retrospective study done at Dr. R. N. Cooper Municipal General Hospital and HBT Medical College. Data collected from January 2018 to January 2020 on 2-year follow-up was available for 31 cases.

Results: Among the cases in which Gene Xpert was used, the sensitivity for the detection of Mycobacterium tuberculosis was 77\% (24/31). Moreover, the sensitivity of Gene Xpert to detect rifampicin resistance was $100 \%(31 / 31)$ in our study. In 11 of the 31 patients $(35 \%)$, diagnosis was confirmed on the basis of a culture. All $31(100 \%)$ patients exhibited a typical magnetic resonance imaging (MRI) picture. Among the 31 patients, 14 patients underwent biopsy, in 21 patients, diagnosis was confirmed on the basis of histopathology (67\%). Smear microscopy was positive in 38\% (12/31) of the patients. ESR was raised in $80 \%$ of the patients and CRP was reactive in $58 \%$ of the patients.

\begin{tabular}{|c|c|}
\hline Test & Sensitivity \\
\hline Gene Expert & $77 \%$ \\
\hline Histopathology & $67 \%$ \\
\hline Smear Microscopy & $38 \%$ \\
\hline Culture & $35 \%$ \\
\hline
\end{tabular}

Conclusions: Gene Xpert is a rapid and highly sensitive tool to diagnose tuberculosis and rifampicin resistance in patients with tuberculosis of the spine. Furthermore, we achieved a $77 \%$ sensitivity for the detection of Mycobacterium tuberculosis and 100\% sensitivity for the detection of rifampicin resistance in our study.

Keywords: Gene Xpert, tuberculosis, spine

\section{Introduction}

Musculoskeletal affection is observed in $4 \%$ of all cases with tuberculosis; $50 \%$ of which involve the spine, which is the most common form of skeletal tuberculosis. ${ }^{1}$ Currently, the diagnosis of tuberculosis of the spine is primarily based on clinico-radiological observations. A typical presentation of tuberculosis of the spine consists of pain during movement with a localized deformity in the back that is tender following percussion as well as other typical systemic symptoms of active tuberculosis (i.e., night cries, malaise, weight loss, loss of appetite, night sweats, and a rise in temperature in the evening). Moreover, patients may or may not have a neurological deficit, which can be the first symptom in rare cases. Microscopic 
confirmation using Ziehl-Nielsen staining remains a popular diagnostic method because of its simplicity and costeffectiveness; however, it has a low sensitivity and requires $10,000-100,000$ bacilli $/ \mathrm{mL}$ in clinical specimens to be positive. Mycobacterium tuberculosis (Mtb) culture is the gold standard method for the diagnosis of tuberculosis, but it also has various limitations, including a required 6-8-week period of growth because of the slow replication rate of the bacteria; these results are often negative as it requires 10-100 bacilli/mL (live bacilli) in clinical specimens to achieve culture positive results. Magnetic resonance imaging (MRI) is a better diagnostic method than radiography. Marrow edema, endplate disruption, paravertebral soft tissue formation, subligamentous collections, and a high signal of the intervertebral disc on T2-weighted are typical MRI features with good to excellent sensitivity for spinal tuberculosis. Overall, the sensitivity and specificity of MRI for spinal tuberculosis are $100 \%$ and $88.2 \%$, respectively. MRI findings with a high sensitivity and specificity include the disruption of the end-plate $(100 \%$ and $81.4 \%$, respectively), paravertebral soft-tissue shadow $(96.8 \%$ and $85.3 \%$, respectively), and high signal intensity of the intervertebral disc on the T2-weighted image $(80.6 \%$ and $82.4 \%$, respectively). Atypical presentation primarily includes discrete foci of spinal involvement with intervening normal vertebrae and no evidence of a connecting soft tissue abscess or any other MRI features typical of tuberculosis as discussed above (i.e., the involvement of only the posterior column of the spine without end plate involvement and multiple skip lesions without a soft tissue shadow). Although the specimen adequacy is higher for an open biopsy than for a percutaneous biopsy, the similarity ratio between the initial radiological and final pathological diagnosis of both techniques are favorable (71.4\% for the open biopsy and $69.2 \%$ for the percutaneous biopsy). ${ }^{9}$ Recent techniques, such as polymerase chain reaction (PCR) and Gene Xpert provide improved accuracy over microscopy and are more rapid than bacterial cultures. The Gene Xpert test has a sensitivity of $95.6 \%$ and a specificity of $96.2 \%$ for diagnosis of spinal tuberculosis.

Additionally, Gene Xpert is more likely to detect Mtb DNA than traditional PCR, with the added advantage of also determining rifampicin resistance. A delay in diagnosis and the failure to detect drug resistance are major hurdles involved in the treatment of tuberculosis of the spine even today.

\section{Materials and Methods}

All cases with tuberculosis of the spine treated at the Dr. R. N. Cooper Hospital (Mumbai), a tertiary care Centre, between 2018 and January 2020 were retrospectively analyzed. Data on a follow-up of 2 years was available for 31 cases. Of these 31 cases, $(77 \%)$ cases were confirmed by Gene Xpert and were included in the present study.

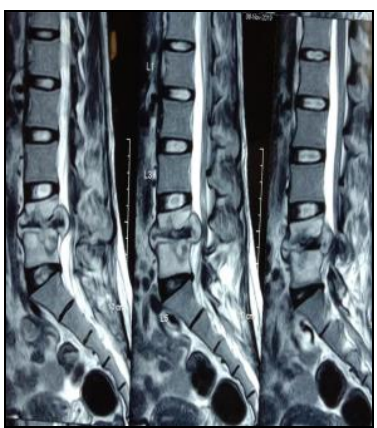

PRE-OP



POST-OP
A typical case of tuberculosis of the spine. A 23-year-old female patient presented with back pain, tenderness in the lumbar spine, and neurological deficit. (A) Typical magnetic resonance imaging picture of tuberculosis of the spine on the T2-weighted image. (B) Diagnosis was established on the basis of an intraoperative tissue sample in this case. The figure shows the postoperative X-ray following surgery.

\section{Results}

In the study 10 male and 21 female patients in the age group of $14-70 y$ rs were included. There were 18 patients who were surgically treated with Decompression and Instrumentation, whereas 13 patients were managed conservatively or with only drainage of abscess. In all of the surgically treated cases, the diagnosis was made on the basis of an intraoperative tissue biopsy obtained from the diseased vertebra and intervening disc space. In the 13 patients managed conservatively, 4 patients underwent a CT guided spinal biopsy while the remaining 9 underwent open abscess drainage.

Gene Xpert was used in 31 cases, in which sample was taken intraoperatively. Of the 24 cases that tested positive using GeneXpert, 1 also tested positive for rifampicin resistance. The patients that tested positive for rifampicin resistance were further confirmed by a drug sensitivity test. Although the sensitivity of Gene Xpert for the detection of rifampicin resistance was $100 \%$ in our study, the overall sensitivity for the detection of MTB was $77 \%$.

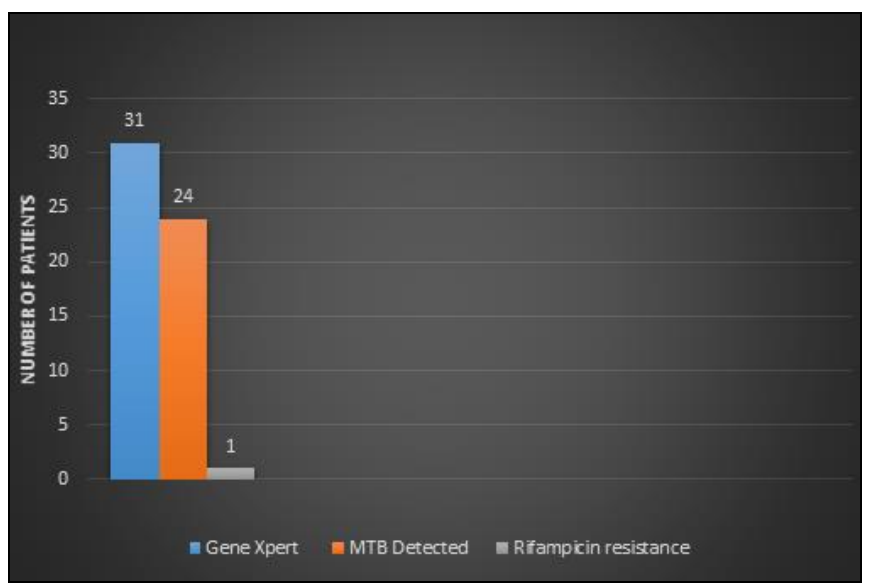

A graph showing whether Gene Xpert was used, if Mycobacterium tuberculosis was detected, the presence of rifampicin resistance (X-axis), and the number of patients ( $\mathrm{Y}$ axis).

\section{Discussion}

A delay in both the diagnosis and initiation of treatment as well as the failure to recognize cases of drug resistance could have an adverse effect on the prognosis of patients with tuberculosis of the spine. Traditional diagnostic methods are based on the typical clinical features followed by a bacteriological confirmation via positive histology and culture. Moreover, the traditional methods of microscopy, histology, and culture have a low sensitivity and specificity. The sensitivity of histology to confirm a diagnosis of spinal tuberculosis has been reported to be approximately $60 \%$. In addition, the incidence of positive cultures for acid-fast bacilli in osteoarticular tuberculous lesions has been reported to be between $40 \%$ and $88 \%$. In our present study, to confirm the diagnosis of tuberculosis of the spine, the sensitivity of the culture was $35 \%$ in the treated cases. Currently, MRI is the 
most popular tool used for the diagnosis of tuberculosis of the spine with good to excellent sensitivity. In addition, Gene Xpert was used in 31 cases. Although the sensitivity of Gene Xpert was very high for tissue samples taken during surgery or open biopsy from diseased vertebra and disc material, the sensitivity was reduced for tissue samples obtained via a percutaneous biopsy. Moreover, the overall sensitivity was $77 \%$, and the sensitivity of Gene Xpert to detect rifampicin resistance was $100 \%$. In all cases Gene Xpert was used to make a rapid and accurate diagnosis. By combining MRI with Gene Xpert, we were able to achieve a considerably high sensitivity for the detection of MTB in cases of tuberculosis of the spine.

\section{Conclusions}

An accurate clinical diagnosis of tuberculosis of the spine requires an extremely high degree of clinical suspicion as one in every three patients can exhibit an atypical clinical presentation. A culture of acid-fast bacilli in osteoarticular tuberculous lesions remains the gold standard diagnostic test, but it is far from being an ideal screening tool to diagnose tuberculosis of the spine because of its low sensitivity. Moreover, MRI is a good screening tool. In contrast, the sensitivity of Gene Xpert for detecting MTB and rifampicin resistance is excellent. In our study, Gene Xpert had a sensitivity of $77 \%$ for detecting MTB and had a sensitivity of $100 \%$ for predicting rifampicin resistance. Combining MRI with Gene Xpert, provides a rapid and highly sensitive diagnosis tool to detect both MTB and rifampicin resistance in patients with tuberculosis of the spine.

\section{References}

1. Tuli SM. Tuberculosis of the skeletal system: bones, joints, spine and bursal sheaths. New Delhi: Jaypee Brothers Pvt. Ltd, 2010.

2. Kumar M, Kumar R, Srivastava AK et al. Sensitivity of PCR IS6110 in relation to culture and staining in Pott's disease. Indian J Neurosurg. 2013; 2:46-51.

3. Singh UB, Bhanu NV, Suresh VN, Arora J, Rana T, Seth P. Utility of polymerase chain reaction in diagnosis of tuberculosis from samples of bone marrow aspirate. Am J Trop Med Hyg. 2006; 75:960-963.

4. Negi SS, Gupta S, Khare S, Lal S. Comparison of various microbiological tests including polymerase chain reaction for the diagnosis of osteoarticular tuberculosis. Indian J Med Microbiol. 2005; 23:245-248.

5. Pandey V, Chawla K, Acharya K, Rao S, Rao S. The role of polymerase chain reaction in the management of osteoarticular tuberculosis. Int Orthop. 2009; 33:801-805.

6. Griffith JF, Kumta SM, Leung PC, Cheng JC, Chow LT, Metreweli C. Imaging of musculoskeletal tuberculosis: a new look at an old disease. Clin Orthop Relat Res. 2002; (398):32-39.

7. Danchaivijitr N, Temram S, Thepmongkhol K, Chiewvit P. Diagnostic accuracy of MR imaging in tuberculous spondylitis. J Med Assoc Thai. 2007; 90:1581-1589.

8. Jain AK, Sreenivasan R, Saini NS, Kumar S, Jain S, Dhammi IK. Magnetic resonance evaluation of tubercular lesion in spine. Int Orthop. 2012; 36:261-269.

9. Yapici F, Atici Y, Balioglu MB, et al. A comparison of two techniques: open and percutaneous biopsies of thoracolumbar vertebral body lesions. J Craniovertebral Junction Spine. 2015; 6:36-39.

10. Held M, Laubscher M, Zar HJ, Dunn RN. GeneXpert polymerase chain reaction for spinal tuberculosis: an accurate and rapid diagnostic test. Bone Joint J. 2014; 96B:1366-1369.

11. Yagmur G, Albayrak N, Das T, Yildirim M, Ozgun A, Buyuk Y. Comparison of two different real-time PCR systems in postmortem diagnosis of tuberculosis in paraffin-embedded tissues. Mikrobiyol Bul. 2014; 48:577-584.

12. Kramer N, Rosenstein ED. Rheumatologic manifestations of tuberculosis. Bull Rheum Dis. 1997; 46:5-8.

13. Van der Spoel van Dijk A, A MC, Botha PL, Shipley JA, Kapnoudhis MA, Beukes CA. The diagnosis of skeletal tuberculosis by polymerase chain reaction. Cent Afr. J Med. 2000; 46:144-149.

14. Agrawal V, Patgaonkar PR, Nagariya SP. Tuberculosis of spine. J Craniovertebr Junction Spine. 2010; 1:74-85.

15. Garg RK, Somvanshi DS. Spinal tuberculosis: a review. J Spinal Cord Med. 2011; 34:440-454. 